Faculdade de Ciências Econômicas UFRGS

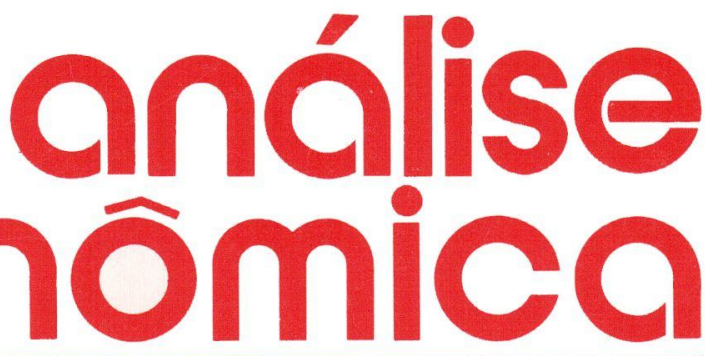

- COMÉRCIO INTERNACIONAL: UMA AGENDA DE PESQUISAS Carlos Alberto Primo Braga

- INFLACÃO E REFORMA MONETÁRIA: KEYNES E MISES Francisco Anuatti

- A ECONOMIA COMO CIÉNCIA Eleutério F.S. Prado

- REAVAlIAÇ̃̃o das PRIVATIZAÇÕES EM PAÍSES EUROPEUS

Márcio Wohlers de Almeida

- INTEGRAÇÃo BRASIL-ARGENTINA E A PRODUÇÃO DE GRÃOS José Eduardo Gutiérrez Perez Walter José Stülp

- SUBSTITUIÇÃO DE COLONOS POR TRABALHADORES ASSALARIADOS

Carlos José Caetano Bacha

- EQUILÍBRIO DISTRIBUTIVO EM MODELOS KALDORIANOS AMPLIADOS Joanílio Rodolpho Teixeira Jorge Thompson Araújo

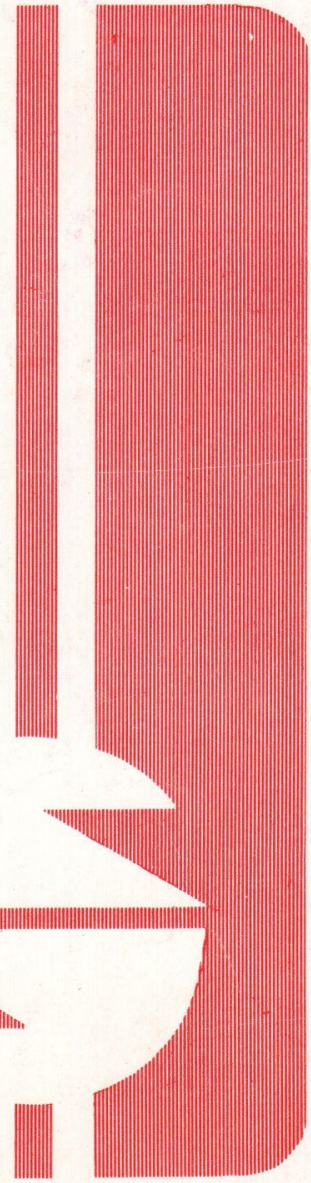


UNIVERSIDADE FEDERAL DO RIO GRANDE DO SUL.

Reitor: Prof. Tuiskon Dick

FACULDADE DE CIËNCIAS ECONÔMICAS

Diretor. Prof. Walter Meucci Nique

CENTRO DE ESTUDOS E PESQUISAS ECONÔMICAS

Diretor. Prof. Reinaldo Ignacio Adams

DEPARTAMENTO DE CIÊNCIAS ECONÔMICAS

Chefe: Prof. Pedro Cezar Dutra Fonseca

CURSO DE PÓS-GRADUAÇĀO EM ECONOMIA

Coordenador. Prof. Roberto Camps Moraes

CURSO DE PÓS-GRADUAÇĀO EM ECONOMIA RURAL

Coordenador. Prot. Aray Miguel Feldens

CONSELHO EDITORIAL:

Achyles Barcelos da Costa, Aray Miguel Feldens, Atos Freitas Grawunder, Carlos Augusto Crusius, Emani Hickmann, João Rogério Sanson, Juvir Luiz Mattuella, Maria Imilda da Costa e Silva; Nali de Jesus de Souza, Nuno Renan Lopes de Figueiredo Pinto, Ot́lia Beatriz Kroeff Carrion, Otto Guilherme Konzen, Paulo Alexandre Spohr, Pedro Cezar Dutra Fonseca, Reinaldo Ignacio Adams, Roberto Camps Moraes, Valter José Stülp, Yeda Rorato Crusius, Edgar Augusto Lanzer (UFSC) e Humberto Vendelino Richeter (UnB)

COMISSĀO EDITORIAL: Atos Freitas Grawunder, Pedro Cezar Dutra Fonseca, Reinaldo Ignacio Adams e Roberto Camps Moraes.

EDITOR: Nali de Jesus de Souza

SECRETARIA: Francisco Carlos Becco dos Santos, Maria Ivone de Mello (normalização), Vanete Ricacheski (revisão de textos), Wagner Nunes e Zélide Bregalda.

FUNDADOR: Prof. Antônio Carlos Santos Rosa

A Revista ANÁLISE ECONÔMICA publica dois números anuais nos meses de março e novembro. O preço da assinatura, para 1990, é de 7,0 BTNs (o número avulso custa 4 BTNs), a ser pago através de cheque nominal em nome da "Faculdade de Ciências Econômicas/ UFRGS". Aceita-se permuta com revistas congêneres. Aceitam-se, também, livros para elaboração de resenhas ou recensōes.

Toda correspondência, material para publicação, assinaturas e permutas devem ser dirigidos ao seguinte destinatário:

PROF. NALI DE JESUS DE SOUZA

Revista Análise Econômica

Av. João Pessoa, 52 - sala 28

90.040 - PORTO ALEGRE (RS) - BRASIL

Telefone: 25-5844 ramal 34 


\title{
EQUILÍBRIO DISTRIBUTIVO EM MODELOS KALDORIANOS AMPLIADOS
}

\author{
Joanílio Rodolpho Teixeira* \\ Jorge Thompson Araújo**
}

\section{INTRODUÇÃO}

Desde a publicação do trabalho pioneiro de Kaldor (1956), a Teoria pós-Keynesiana tem se enriquecido enormente. Seguindo a tradição clássica, ele trata da distribuição da renda $(Y)$ a partir da divisão da mesma entre as classes sociais - trabalhadores e capitalistas - que recebem, respectivamente, salários (W) e lucros (II). Ele introduz no modelo um mecanismo de ajustamento que consiste em duas taxas de poupanças, uma dos trabalhadores $\left(s_{w}\right)$ e outra dos capitalistas ( $\left.s_{\Pi}\right)$. Mostra então que, sob certas condições, a participação dos investimentos na renda $(I / Y)$ determina a participação dos lucros na mesma (II/Y) e, portanto, a poupança dos capitaliştas $\left(\mathrm{S}_{\Pi}\right)$.

Embora Kaldor tenha incorporado na abordagem a poupança dos trabalhadores $\left(S_{w}\right)$, ele deixou de contemplar a possibilidade dos mesmos utilizarem a mesma para adquirir parcela do capital $(K)$ e, portanto, não analisou os efeitos decorrentes. Pasinetti (1962) corrigiu essa omissão e mostrou que, na trajetória de crescimento de longo prazo, se a taxa de juros é igual a taxa de lucro, a propensão a poupar dos trabalhadores não influencia a distribuição de renda entre capitalistas e trabalhadores. Esse resultado teórico é chamado por Pasinetti (1989) de "Teorema de Cambridge". Ele conclui que a taxa de lucro é determinada pela taxa natural de crescimento dividida pela propensão dos capitalistas a poupar. Tal conclusão independe da tecnologia e da propensão a poupar dos trabalhadores.

* Professor do Departamento de Economia de Brasflia - UnB.

** Aluno do Curso de Mestrado em Economia - UnB.

\begin{tabular}{|l|l|l|l|l|} 
ANÁLISE ECONÔMICA & ANO 7 & No 12 & NOVEMBRO/89 & p.139-149 \\
\hline
\end{tabular}


Nos propomos a estender os resultados acima em diversas direções. Reelaboramos o modelo, tomando como inspiração Steedman (1972) e Pasinetti (1989) que, entre outras coisas, incorporaram o papel efetivo do governo, através da tributação e dispêndio público. Na nossa primeira versão, tratamos do equilíbrio distributivo para uma economia fechada com apenas tributação direta. Estudamos também as condições de estabilidade local. Na segunda situação, abrimos o modelo para a economia internacional, mantendo a hipótese de existência de apenas tributação direta. Na terceira versão do modelo, estudamos o caso de uma economia fechada com tributação direta e.indireta. Finalmente, na quarta situação, partimos para o caso de uma economia aberta com tributação direta e indireta. Em todas as versões do modelo, discutimos as condições da estabilidade.

\section{VERSÕES ALTERNATIVAS E ESTENDIDAS DO MODELO}

Steedman (1972), tanto quanto saibamos, foi o primeiro a incorporar o papel efetivo do governo na abordagem Kaldoriana. Ele mostrou que a tributação não exerce qualquer influência sobre a taxa de lucro Ifquido. Contudo, ele deixou de considerar o estoque de capital nas mãos do governo. Ele se concentrou, como Kaldor (1956) e Pasinetti (1962) e (1989), na questão distributiva e na taxa de lucro de longo prazo da economia como um todo. O passo fundamental para se obter a última, bem como se chegar a uma distribuição de longo prazo para a renda nacional reside na explicitação das componentes da função poupança.

\section{1: SITUAÇÃO: Economia Fechada com Tributação Direta}

Sejam $t_{W}$ e $t_{I I}$, respectivamente, as cargas tributárias liquidas sobre a renda-salário e renda-lucro, onde $0 \leqslant t_{w} \leqslant 1 ; 0<t_{\Pi}<1 \mathrm{e}$ $t_{\Pi}>t_{w}$. Nesse caso, $T=t_{w} W+t_{\Pi} \Pi$ onde $T$ é a arrecadação tributária líquida.

Tendo em vista que o estoque de capital da economia como um todo é decomposto em capital dos trabalhadorés $\left(K_{W}\right)$, dos capitalistas $\left(K_{\Pi}\right)$ e do governo $\left(K_{g}\right)$ e que os mesmos geram fluxos de renda $\Pi_{w}, \Pi_{\Pi}$ e $\Pi_{g}$, respectivamente, podemos definir as funções poupança. Sejam $S$ a poupança agregada, $S_{W}$ a dos trabalhadores, $S_{\Pi}$ a dos capi- 
talistas $e \mathrm{~S}_{\mathrm{g}}$ a do governo. Sejam $\mathrm{s}_{\mathrm{w}}$, $\mathrm{s}_{\Pi}$ e $\mathrm{s}_{\mathrm{g}}$ as respectivas taxas de poupança, onde $0 \leqslant s_{w}<1 ; 0 \leqslant s_{w} \leqslant 1$. Suponhamos tambérn que existe uma única carga tributária lfquida sobre a renda-lucro $\left(\mathrm{t}_{\Pi}\right)$ independentemente de sua apropriação pelos capitalistas ou pelos trabaIhadores. Nesse caso teríamos:

(1) $S=S_{W}+S_{I I}+S_{g}$;

(2) $s_{w}=s_{w}\left(1-t_{w}\right) w+s_{w}\left(1-t_{\Pi}\right) \Pi_{w}$;

(3) $s_{\Pi}=s_{\Pi}\left(1-t_{\Pi}\right) \Pi_{\Pi}$;

(4) $s_{g}=s_{g}\left(t_{\Pi} \Pi_{\Pi}+t_{\Pi} \Pi_{w}+t_{w} w+\Pi_{g}\right)=s_{g}\left(T+\Pi_{g}\right)$.

Substitui-se (2), (3) e (4) em (1), obtém-se:

(5) $S=\left[s_{w}\left(1-t_{w}\right)+s_{g}\left(t_{w}\right)\right] w+\left[s_{w}\left(1-t_{\Pi}\right)+s_{g}\left(t_{\Pi}\right)\right] \Pi_{w}+$ $+\left[s_{\Pi}\left(1-t_{\Pi}\right)+s_{g} t_{\Pi}\right] \Pi_{\Pi}+s_{g} \Pi_{g}$

Definindo-se as propensões a poupar "corrigidas" pela tributação:

(6) $s_{w w}^{\prime}=s_{w}\left(1-t_{w}\right)+s_{g} t_{w}$;

(7) $s_{w} \Pi=s \cdot\left(1-t_{\Pi}\right)+s_{g} t_{\Pi}$;

(8) $s_{\Pi}^{\prime}=s_{\Pi}\left(1-t_{\Pi}\right)+s_{g} t_{\Pi}$.

De modo que:

(9) $s=s_{w w}^{\prime} \cdot w+s_{w}^{\prime} \Pi \cdot \Pi_{w}+s_{\Pi}^{\prime} \cdot \Pi_{\Pi}+s_{g} \cdot \Pi_{g}$. 
A taxa de lucro a longo prazo pode ser obtida a partir das seguintes hipóteses (Cf. Pasinetti, 1962 e 1989):

(i) $S / K=S_{w} / K_{w}=S_{\Pi} / K_{\Pi}=S_{g} / K_{g}$;

(ii) $\mathrm{I}=\mathrm{S}$;

(iii) $\Pi / K=\Pi_{w} / K_{w}=\Pi_{\Pi} / K_{\Pi}=\Pi_{g} / K_{g}=r$.

De (ii): $1=S \Rightarrow I / K=S / K$.

De (i): $\frac{1}{k}=\frac{s_{\Pi}}{k_{\Pi}} \Rightarrow \frac{1}{k}=s_{\Pi} \cdot \frac{\Pi_{\Pi}}{k_{\Pi}} \Rightarrow \frac{\Pi_{\Pi}}{k_{\Pi}}=\frac{1}{s_{\Pi}^{\prime}} \cdot g_{n} \cdot$

(10) De (iii): $r=\frac{\Pi}{\mathrm{K}}=\frac{\Pi_{\Pi}}{\mathrm{K}_{\Pi}}=\frac{1}{\mathrm{~s}_{\Pi}^{3}} \cdot \mathrm{g}_{\mathrm{n}}=\frac{1}{\mathrm{~s}_{\Pi}^{\prime}\left(1-\mathrm{t}_{\Pi}\right)+\mathrm{s}_{\mathrm{g}} \mathrm{t}_{\Pi}} \cdot \mathrm{g}_{\mathrm{n}}$.

Expressão que corresponde à taxa de lucro. (onde $g_{n}$ é a taxa natural do crescimento).

A distribuição de renda a longo prazo é obtida diretamente de $r$ :

(11)

$$
\begin{aligned}
\frac{\Pi I}{K} & =\frac{I I}{Y} \cdot \frac{Y}{K} \Rightarrow \frac{\Pi}{Y} \cdot \frac{\Pi}{K}=\frac{1}{s_{\Pi}^{\prime}} \cdot \frac{1}{K} \Rightarrow \frac{\Pi}{Y}=\frac{1}{s_{\Pi}^{\prime}} \cdot \frac{1}{Y}= \\
& =\frac{1}{s_{\Pi}^{\prime}\left(1-t_{\Pi}\right)-s^{t} \Pi} \cdot \frac{1}{Y} \cdot
\end{aligned}
$$

A condição de estabilidade do modelo, extrailda a partir de uma função excesso de demanda "a la Samuelson", é dada por Pasinetti (1962):

$$
\frac{d(S / Y)}{d(\Pi / Y)}>0
$$

Logo,

$$
\frac{d}{d(\Pi / y)}\left\{\left[s_{w}\left(1-t_{w}\right)+s_{g} t_{w}\right] \frac{W}{Y}+\left[s_{w}\left(1-t_{\Pi}\right)+s_{g} t_{\Pi}\right] \frac{I_{w}}{Y}+\right.
$$




$$
\left.+\left[s_{\Pi}\left(1-t_{\Pi}\right)+s_{g} t_{\Pi}\right] \frac{\Pi_{\Pi}}{Y}+s_{g} \frac{\Pi_{g}}{Y}\right\}>0
$$

No que concerne à inequação (13), a única derivada a respeito da qual pode-se ter alguma segurança é $d(W / Y) / d(\Pi / Y)$, igual a -1. Expressando-se $\Pi_{\Pi}$ em termos de $\Pi, \Pi_{w}$ e $\Pi_{g}$, restariam duas derivadias (em principio indefinidas), necessitando de hipóteses adicionais para $d\left(\Pi_{w} / Y\right) / d(\Pi / Y)$ e d $\left(\Pi_{g} / Y\right) / d(\Pi / Y)$.

\section{SITUAÇÃO: Economia Aberta com Tributação Direta}

Neste modelo, a poupança total é desdobrada em poupança externa $\theta$ poupança interna $\left(S_{i}\right)$. Sejam $X$ e $M$, respectivamente, as exportaç̄es e importações. Nesse caso temos:
(1) $S=S+H$
(poupança total).
(2) $s_{i}=s_{w}+s_{\Gamma j}+S g$
(poupança interna).
(3) $H=M-X$
(hiato de recursos reais).

(Obs: as equações de definição de $S_{g}, S_{\Pi I}$ e $S_{g}$ permanecem as mesmas do caso anterior).

Consequientemente, (1) pode ser reescrita como:

(4) $S=s_{w w}^{\prime} W+s_{w}^{\prime} \Pi \Pi_{w}+s_{\Pi}^{\prime} \cdot \Pi_{\Pi}+s_{g} \Pi_{g}+M-X$.

A taxa de lucro pode ser obtida pelo mesmo processo da situação anterior:

$$
\begin{aligned}
\mathrm{I}=\mathrm{S} \Rightarrow \frac{1}{\mathrm{~K}}=\frac{\mathrm{S}}{\mathrm{K}}=\frac{\mathrm{S}_{\Pi}}{\mathrm{K}_{\Pi}} \Rightarrow \mathrm{S}_{\Pi}=\mathrm{s}_{\Pi}^{\prime} \cdot \frac{\Pi_{\Pi}}{\mathrm{K}_{\Pi}} \\
\quad \Rightarrow s_{\Pi}^{\prime} \cdot \frac{\Pi_{\Pi}}{\mathrm{K}_{\Pi}}=\frac{1}{\mathrm{~K}} \Rightarrow \frac{\Pi_{\Pi}}{\mathrm{K}_{\Pi}}=\frac{1}{\mathrm{~s}_{\Pi}^{\prime}} \cdot \mathrm{g}_{\mathrm{n}} \Rightarrow
\end{aligned}
$$


(5) $\frac{\Pi}{\mathrm{K}} \cdot \frac{1}{\mathrm{~s}_{\Pi}^{\prime}} \cdot g_{n}=\frac{1}{\mathrm{~s}_{\Pi}^{\prime}\left(1-\mathrm{t}_{\Pi}\right)+\mathrm{s}_{\mathrm{g}} \mathrm{t}_{\Pi}} \cdot g_{n}$, taxa de lucro.

A participação dos lucros na renda nacional novamente será:

(6) $\frac{\Pi}{Y}=\frac{1}{s_{\Pi}^{\prime}\left(1-t_{\Pi}\right)+s_{g} t_{\Pi}} \cdot \frac{1}{Y}$.

Como (5) e (6) são exatamente iguais às equações (10) e (11) da primeira situação, aparentemente, a introdução da hipótese de economia aberta não afeta o equilíbrio distributivo a longo prazo. Entretanto, isto não descarta a possibilidade de modificações na função poupança dos capitalistas, a partir da qual foram calculadas (5) e (6), advindas da incorporação de variáveis de comércio internacional, as quais poderiam nos levar a conclusões distintas.

De qualquer modo, a condição de estabilidade dinâmica do equilibrio é modificada:

(7) $\frac{d}{d(\Pi / Y)}\left\{\left[s_{w}\left(1-t_{w}\right)+s_{g} t_{w}\right] \frac{W}{Y}+\left[s_{w}\left(1-t_{\Pi}\right)+s_{g} t_{\Pi}\right] \frac{\Pi_{w}}{Y}+\right.$
$\left.+\left[s_{\Pi}\left(1-t_{\Pi}\right)+s t_{\Pi}\right] \frac{\Pi_{\Pi}}{Y}+s_{g} \frac{\Pi_{g}}{Y}+\frac{M}{Y}-\frac{x}{Y}\right\}>0$.

Com efeito, haveria mais duas variáveis indefinidas: $d(M / Y) / d(\Pi / Y)$ e $d(X / Y) / d(\Pi / Y)$.

\section{3ª SITUAÇÃO: Economia Fechada com Tributação Direta e Indireta}

Esta situação é a que mais se aproxima da considerada por Pasinetti (1989), com a única diferença de que, aqui, o governo é co-proprie- 
tário do estoque de capital existente no setor público, dele extraindo urn lucro $\Pi_{\mathrm{g}}$.

A inclusão da tributação (indireta) sobre o consumo, inclusive o governamental, permite uma expressão mais ampla para a "destruição de poupanças" pelo governo. Na primeira situaçäo, esta poderia ser vista nos componentes $s_{g} t_{\Pi}$ e $s_{g} t_{w}$ das equações (6) a (8).

As funções poupança de cada segmento da sociedade, ainda não corrigidas pela "destruição de poupanças" pelo governo, mantêm o mesmo formato apresentado nas equações (2), (3) e (4) da primeira situação. Por conseguinte, a função poupança total continua expressa corno:

(1) $S=S_{w}+S_{\Pi}+S_{g}$.

Há duas novas igualdades a serem introduzidas. A primeira, dada pela equação (2), mostra o consumo do governo, e a segunda, representada por (3), fornece a receita tributária do governo acrescida de uma aliquota única $\mathrm{t}_{\mathrm{j}}$. Esta incide sobre o consumo dos trabalhadores, capitalistas e do próprio governo, constituindo, portanto, em tributação indireta. ${ }^{1}$

(2) $C_{g}=\left(1-s_{g}\right)\left(\Pi_{g}^{*}+T\right)$, onde $\Pi_{g}^{*}=\left\{1-\left[t_{i}\left(1-s_{g}\right)\right]\right\} \Pi_{g}$

(3) $T=\left[t_{w} \cdot w+t_{\Pi} \Pi_{w}+t_{\Pi} \Pi_{\Pi}\right]+$

$+t_{i}\left[\left(1-s_{w}\right)\left(1-t_{w}\right) w+\left(1-s_{w}\right)\left(1-t_{\Pi}\right) \Pi_{w}+\left(1-s_{\Pi}\right)\left(1-t_{\Pi}\right) \Pi_{\Pi}+c_{g}\right]$

Substituindo (2) em (3):

$T=\left[t_{w}+t_{j}\left(1-s_{w}\right)\left(1-t_{w}\right)\right] w+\left[t_{\Pi}+t_{i}\left(1-s_{w}\right)\left(1-t_{\Pi}\right)\right] \Pi_{w}+$

$+\left[t_{I I}+t_{i}\left(1-s_{\Pi I}\right)\left(1-t_{\Pi}\right)\right] \Pi_{\Pi}+t_{i}\left(1-s_{g}\right) \Pi_{g}^{*}+t_{i}\left(1-s_{g}\right) T$.

Sendo $a=\left[1-t_{i}\left(i-s_{g}\right)\right]-1$ obtemos:

(4) $T=a \quad\left[t_{w}+t_{i}\left(1-s_{w}\right)\left(1-t_{w}\right)\right] w+\left[t_{\Pi}+t_{i}\left(1-s_{w}\right)\left(1-t_{\Pi}\right)\right] \Pi_{w}+$

1 Como a parcela indireta jå inclui uma parcela dos lucros do setor público, por intemédio da alfquota $\hbar_{\text {, }}$ que incide tambêm sobre o consumo governarnental, definimos a nova variável $\Pi_{g}$, de modo a evitarmos a dupla contagem da referida parcela. 
$+\left[t_{\Pi} \div t_{i}\left(1-s_{\Pi}\right)\left(1-t_{\Pi}\right)\right] \Pi_{\Pi}+t_{i}\left(1-s_{g}\right) \Pi_{g}^{*}$.

A função poupança do governo, por conseguinte, será dada por.

(5) $s_{g}=s_{g} a\left\{\left[t_{w}+t_{i}\left(1-s_{w}\right)\left(1-t_{w}\right)\right] w+\left[t_{\Pi}+t_{i}\left(1-s_{w}\right)\left(1-t_{\Pi}\right)\right] \Pi_{w}+\right.$ $+\left[t_{\Pi}+t_{i}\left(1-s_{\Pi)}\left(1-t_{\Pi}\right)\right] \Pi \Pi\right\}+s_{g} a t_{i}\left(1-s_{g}\right) \Pi_{g}^{*}+s_{g} \Pi_{g}^{*}$

Alternativamente, $S_{g}$ pode ser expressa como:

(6) $s_{g}=s_{g} a\left\{\left[t_{w}+t_{j}\left(1-s_{w}\right)\left(1-t_{w}\right)\right] w+\left[t_{\Pi}+t_{i}\left(1-s_{w}\right)\left(1-t_{\Pi}\right)\right] \Pi_{w}+\right.$ $+\left[t_{\Pi}+t_{j}\left(1-s_{\Pi}\right)\left(1-t_{\Pi}\right) \Pi \Pi\right\}+s_{g} \Pi_{g}\left\{(a-1)\left[t_{j}\left(1-s_{g}\right)+1\right]\right\}$

Portanto, a função poupança total será dada por:

(7) $s=s_{w w}^{\prime \prime} w+s_{w \Pi}^{\prime \prime} \Pi_{w}+s_{\Pi}^{\prime \prime} \Pi_{\Pi}+s_{g} \Pi_{g}\left\{(a-1)\left[t_{i}\left(1-s_{g}\right)+1\right]\right\}$ onde:

(8) $s_{w w}^{\prime \prime}=s_{w}\left(1-t_{w}\right)+s_{g} a\left[t_{w}+t_{i}\left(1-s_{w}\right)\left(1-t_{w}\right)\right]$;

(9) $s_{w I I}^{\prime \prime}=s_{w}\left(1-t_{\Pi}\right)+s_{g} a\left[t_{\Pi I}+t_{i}\left(1-s_{w}\right)\left(1-t_{\Pi}\right)\right.$;

(10) $s_{\Pi}^{\prime \prime}=s_{\Pi}\left(1-t_{\Pi}\right)+s_{g} a\left[t_{\Pi}+t_{j}\left(1-s_{\Pi}\right)\left(1-t_{\Pi}\right)\right]$.

A taxa de lucro de longo prazo, obtida pelo mesmo método das situações anteriores, é dada por:

$$
\frac{\Pi}{k}=\frac{1}{s_{\Pi}\left(1-t_{\Pi}\right)+s_{g} a\left[t_{\Pi}+t_{i}\left(1-s_{\Pi}\right)\left(1-t_{\Pi}\right)\right]} g_{n}=\frac{1}{s_{\Pi}^{n}} g_{n}
$$

De (11), a participação dos lucros (de longo prazo) na renda nacional pode ser obtida diretamente:

$$
\frac{\Pi}{Y}=\frac{1 / Y}{s_{\Pi}\left(1-t_{\Pi}\right)+s_{g} a\left[t_{\Pi}+t_{i}\left(1-s_{\Pi}\right)\left(1-t_{\Pi}\right)\right]}=\frac{1}{s_{\Pi}^{n}} \cdot \frac{1}{Y}
$$

O equilíbrio será dinamicamente estável se: 
$\frac{d}{d(\Pi / Y)}\left\{s_{w w}^{\prime \prime} \frac{W}{Y}+s_{w \Pi}^{\prime \prime} \frac{\Pi w}{Y}+s^{\prime \prime} \Pi \frac{\Pi_{\Pi}}{Y}+s_{g}(a-1)\left[t_{i}\left(1-s_{g}\right)+1\right]\right\}$

Mais uma vez, a condição de estabilidade dependerá do comportamento das derivadas $d\left(\Pi_{w} / Y\right) / d(\Pi / Y)$ e $d\left(\Pi_{W} / Y\right) / d(\Pi / Y)$, exigindo hipóteses adicionais.

\section{4․ SITUAÇÃO: Economia Aberta com Tributação Direta e Indireta.}

A modificdção fundamental decorrente da hipótese de economia aberta manifesta-se sobre a função poupança total, desdobrada em poupança interna $\left(S_{i}\right)$ e hiato de recursos reais $(H)$. Como vimos na segunda situação:

(1) $S=S_{i}+H$

A função $S_{i}$, agora, corresponde à função $S$ na terceira situação:

(2) $s_{i}=s_{w w}^{n} w+s_{w}^{\prime \prime} \Pi_{w}+s_{\Pi}^{\prime \prime} \Pi_{\Pi}+s_{g}\left[(a-1) t_{i}\left(1-s_{g}\right)+1\right] \Pi_{g}$.

Por outro lado, $\mathrm{H}$ é o saldo do balanço de pagamentos em transações correntes multiplicado por -1:

(3) $H=M-X$.

A introdução de $\mathrm{H}$, definido como em (3), em nada afeta o equilibrio distributivo, já que:

(4) $\frac{\mathrm{II}}{\mathrm{K}}=\frac{1}{\mathrm{~s}^{\mu} \Pi} g_{\mathrm{n}}$

(5) $\frac{\Pi}{Y}=\frac{1}{s_{\Pi}^{n}} \frac{1}{Y}$

e 
Da mesma forma que, na segunda situação, a introdução de $\mathrm{H}$ altera a condição de estabilidade do equilibrio:

(6)

$$
\begin{aligned}
\frac{d}{d(\Pi / Y)} \quad & \left\{s^{\prime \prime} w \frac{W}{Y}+s^{\prime \prime} w w \Pi \frac{\Pi_{w}}{Y}+s^{\prime \prime} g \frac{\Pi_{\Pi}}{Y}+\right. \\
& \left.+s_{g}\left[(a-1) t_{i}\left(1-s_{g}\right)+1\right] \frac{\Pi_{g}}{Y}+\frac{M}{Y}-\frac{X}{Y}\right\}>0 .
\end{aligned}
$$

Portanto, há quatro derivadas exigindo hipóteses adicionais: $d\left(\Pi_{W} / Y\right) /(d(\Pi / Y)$, $d(X / Y) / d(\Pi / Y)$.

\section{3 - CONCLUSÕES}

Verificamos, assim, que é possivel estender o modelo de KaldorPasinetti, da taxa de lucro e distribuição da renda em relação ao crescimento, em diversas direções. Foram mostradas quatro versões do modelo: I) economia fechada com apenas tributação direta; II) economia aberta com tributação direta; III) economia fechada com tributação direta e também indireta; IV) economia aberta com tributação direta e indireta.

Não há dificuldade formal em se estabelecer, para o longo prazo, tanto a taxa de lucro quanto a participação dos lucros na renda nacional, para cada uma das extensões propostas. No entanto, nos quatro casos analisados, não fica clara a questão da estabilidade. Isso decorre do fato de que algumas das derivadas relevantes têm sinais indefinidos. São, portanto, necessárias algumas hipóteses adicionais para garantir a estabilidade, de longo prazo, no comportamento dos modelos alternativos. É interessante ressaltar que a introdução da poupança externa, desde que não seja colocada como função de algum tipo de taxa de juros, em nada afeta o equilíbrio distributivo já obtido com uma economia fechada, mas apenas as condições de estabilidade do equilíbrio a longo prazo.

\section{BIBLIOGRAFIA}

KALDOR, N. Alternative Theories of Distribution, Review of Economic Studies, vol. 23, 1956. 
PASINETTI, L. Rate of Profit and Income Distribution in Relation to the Rate of Economic Growth, Review of Economic Studies, vol. 29, 1962.

- Ricardian Debt/Taxation Equivalence in the Kaldor Theory of Profits and Income Distribution, Cambridge Journal of Economics, vol. 13, 1989.

STEEDMAN, I. The State and the Outcome of the Pasinetti Process, Economic Journal, vol. LXXXII, 1972. 